\title{
Acute In-Stent Thrombosis after Carotid Angioplasty and Stenting: A Case Report and Literature Review
}

\author{
Wei Hu Li Wang GuoPing Wang \\ Department of Neurology, Affiliated Provincial Hospital of Anhui Medical University, \\ Hefei, PR China
}

\section{Keywords}

Carotid artery disease $\cdot$ Stent restenosis $\cdot$ Revascularization $\cdot$ Arterial thrombolysis $\cdot$ Balloon dilation

\begin{abstract}
Background: Based on the results of a recent randomized controlled trial, carotid artery stenting (CAS) was regarded as a relatively safe, less invasive treatment of internal carotid artery stenosis. However, cerebral thromboembolic events are the most common complications of CAS. Especially acute stent thrombosis following CAS will be fatal without prompt diagnosis and revascularization. Case Report: We report a case of acute stent thrombosis in whom carotid revascularization was performed successfully via arterial thrombolysis and balloon postdilation. A 79-year-old man with hypertension was hospitalized for an episode of transient ischemic attack. Computed tomography angiography revealed subtotal occlusion in the left carotid artery. Aspirin (100 mg) and clopidogrel $(75 \mathrm{mg}$ ) were administered daily for 5 days before the procedure. CAS was performed under local anesthesia. The first postprocedural angiogram showed the stent looked good. However, a repeat angiogram showed in-stent thrombosis 2 min after withdrawal of the cerebral protection filter. Interestingly, the patient presented no neurologic deficit. After an additional 2,000 U of heparin had been administered intravenously, a microcatheter (SL-14; Boston Scientific, USA) was positioned to the in-stent thrombosis. Next, a total dose of $10 \mathrm{mg}$ of recombinant tissue plasminogen activator was injected into the thrombus via the microcatheter within $10 \mathrm{~min}$, which led to partial recanalization with antegrade flow. However, complete occlusion of the lesion occurred 5 min later. Under the guidance of angiography roadmap, a protection filter (Emboshield NAV6; Abbott Vascular, USA) was deployed at the distal part of the stent and redilation of the stent was per-
\end{abstract}

W. Hu and L. Wang contributed equally to this work. 
formed with a $5 \times 30 \mathrm{~mm}$ balloon (Viatrac 14 Plus; Abbott Vascular) at $14 \mathrm{~atm}$. Finally, carotid revascularization was performed successfully, proven by postprocedural angiogram. Conclusion: Acute carotid stent thrombosis (ACST) can have devastating effects on the survival of the patient. For ACST when the stent does not fully adhere to the blood vessel, a mechanical approach should be a feasible solution to the problem.

(c) 2018 S. Karger AG, Basel

\section{Introduction}

Based on the results of a recent randomized controlled trial, carotid artery stenting (CAS) was regarded as a relatively safe, less invasive treatment of internal carotid artery stenosis [1]. Similar to other treatments for internal carotid artery stenosis, cerebral thromboembolic events are the most common complications of CAS. Especially acute stent thrombosis following CAS will be fatal without prompt diagnosis and revascularization.

Here, we reported a case of acute stent thrombosis in whom carotid revascularization was performed successfully via arterial thrombolysis and balloon postdilation. The related publications were reviewed to assess the feasibility of vascular recanalization.

\section{Case Report}

A 79-year-old man with hypertension was hospitalized for an episode of transient ischemic attack, which was etiologically attributed to severe left internal carotid artery stenosis. Two months before, he had been admitted to a local hospital with transient right-sided hemiparesis. Aspirin (100 mg), together with atorvastatin $(20 \mathrm{mg})$, were orally administered daily. On the onset of the event, a left cerebral hemisphere infarct was shown by brain magnetic resonance imaging. Computed tomography angiography revealed subtotal occlusion of the left carotid artery (Fig. 1) and approximately $60 \%$ stenosis in the contralateral carotid bifurcation. In 2007, the patient was fully recovered from the first-line treatment, and informed consent was obtained from the patient.

Aspirin (100 mg) and clopidogrel (75 mg) were administered daily 5 days before the procedure. CAS was performed under local anesthesia. Intraoperatively, heparin was administered intravenously at a dose of 5,000 U (100 U/kg) bolus immediately after femoral artery puncture and at a dose of 1,000 U/h during the

Fig. 1. Severe left internal carotid stenosis on computed tomography angiography.

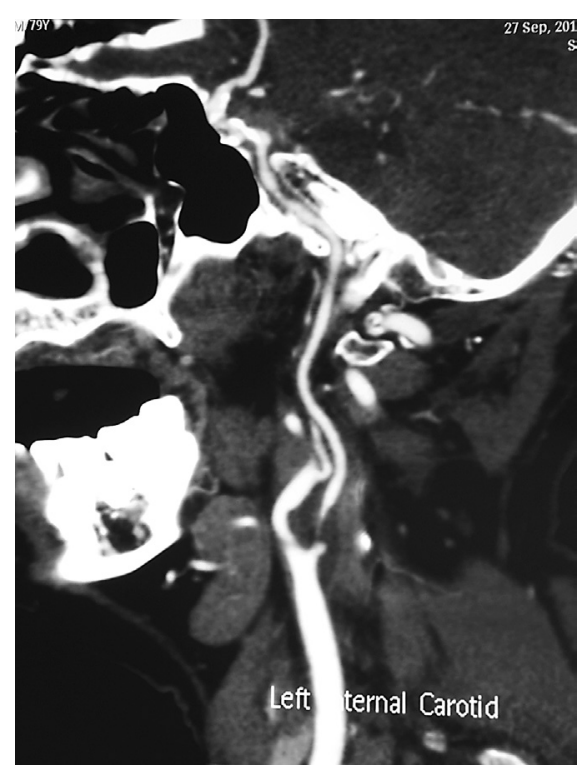


rocedure. Angiography revealed almost complete left carotid artery occlusion (Fig. 2a). After a cerebra protection filter (spider FX; EV-3, USA) had been positioned, the stenosis of the left internal carotid artery was predilated with a $3 \times 20 \mathrm{~mm}$ balloon (Savvy; Cordis, The Netherlands) and a $4 \times 30 \mathrm{~mm}$ balloon (Viatrac 14 Plus; Abbott Vascular, USA). An $8 \times 30 \mathrm{~mm}$ self-expandable carotid stent (Xact; Abbott Vascular) was then implanted and released using a 0.014-inch guidewire and a 6-F guide catheter. The first postprocedural angiogram showed the carotid stent was not well and dilated by a $5 \times 20 \mathrm{~mm}$ balloon at $10 \mathrm{~atm}$ (Fig. $2 \mathrm{~b}$ ). However, a repeat angiogram showed in-stent thrombosis $2 \mathrm{~min}$ after withdrawal of the cerebral protection filter (Fig. 3). Interestingly, the patient presented no neurologic deficit. After an additional 2,000 U of heparin had been administered intravenously, a microcatheter (SL-14; Boston Scientific, USA) was positioned to the in-stent thrombosis. Next, a total dose of $10 \mathrm{mg}$ of recombinant tissue plasminogen activator was injected into the thrombus via the microcatheter within $10 \mathrm{~min}$, which led to partial recanalization with antegrade flow (Fig. 4). However, complete occlusion of the lesion occurred $5 \mathrm{~min}$ after carotid thrombolysis according to the repeat angiogram (Fig. 5). Under the guidance of angiography roadmap, a protection filter (Emboshield NAV6; Abbott Vascular) was deployed at the distal part of the stent, and redilation of the stent was performed with a $5 \times 30 \mathrm{~mm}$ balloon (Viatrac 14 Plus) at $14 \mathrm{~atm}$. Finally, carotid revascularization was performed successfully, proven by postprocedural angiogram (Fig. 6a, b).

A thromboelastography test showed that the patient with the gene CYP2C19 had a normal response to clopidogrel. Clopidogrel $(75 \mathrm{mg})$ and atorvastatin $(20 \mathrm{mg})$ were administered daily. At the 10-month followup period, the patient showed no signs of neurologic deficits, without any evidence of restenosis or occlusion after CAS proven by duplex ultrasonography (Fig. 7).

\section{Discussion and Literature Review}

Acute carotid stent thrombosis (ACST) is a very rare complication of CAS and can have devastating effects on patient survival. The probable factors contributing to ACST include systemic inadequate antiplatelet treatment, clopidogrel hyporesponse, local vessel dissection, a hypercoagulable state, severe plaque protrusion, vasospasm, intimal injury, stent thrombogenicity, early stent restenosis, and stent underexpansion [2].
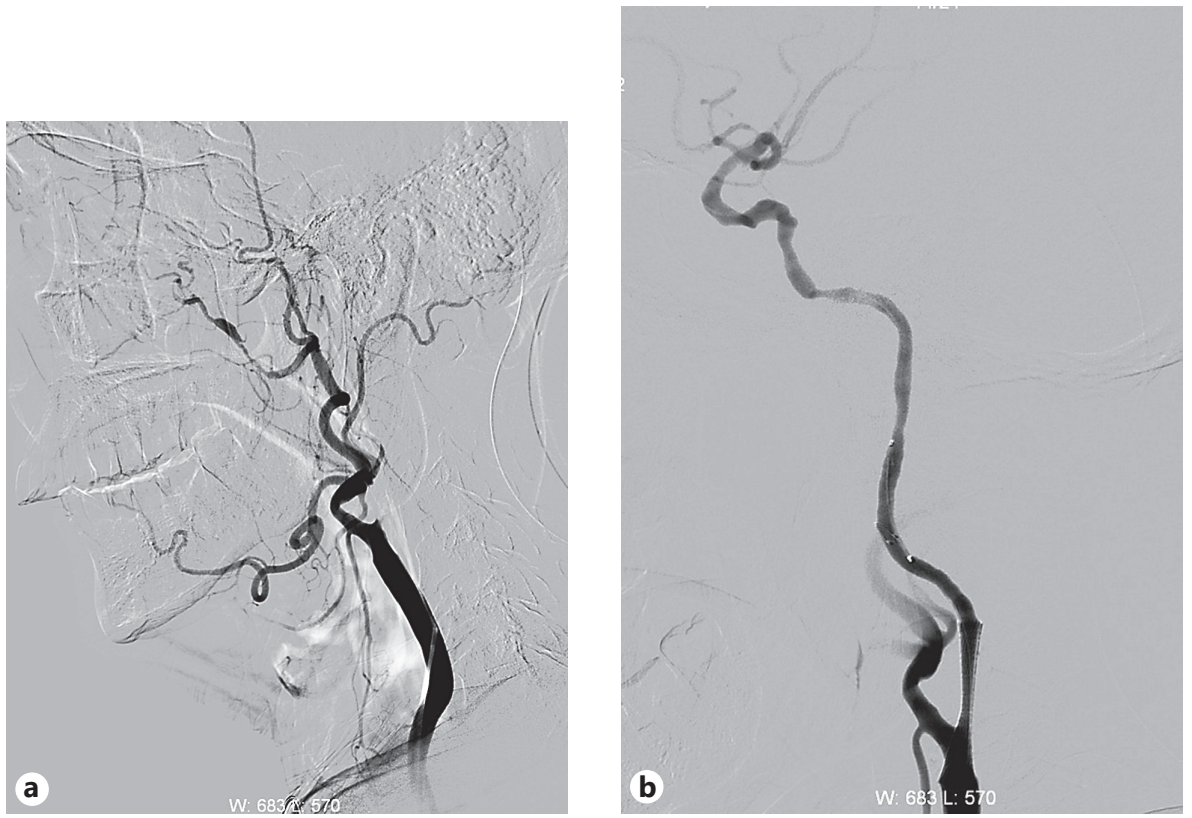

Fig. 2. Severe internal carotid stenosis before (a) and after the successful carotid artery stenting procedure (b). 
Interventional

Neurology

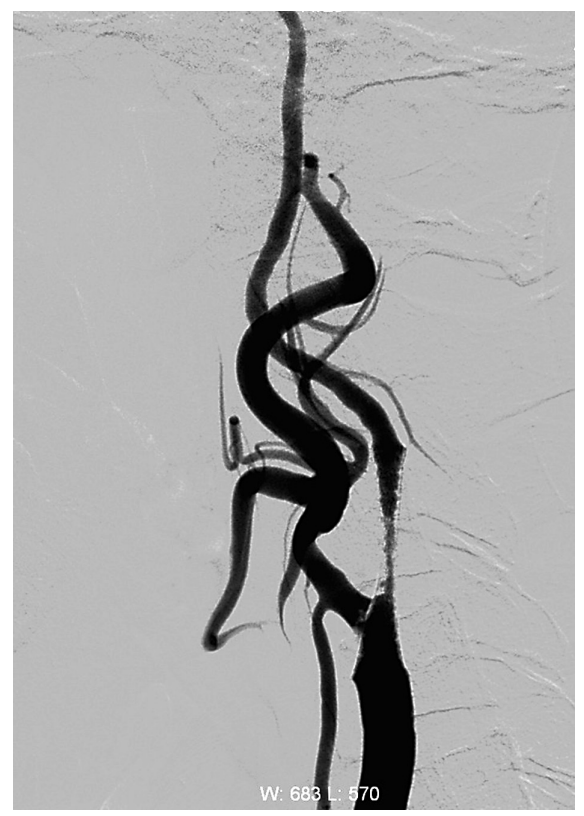

Fig. 3. Acute carotid stent thrombosis as confirmed by angiography.

\begin{tabular}{l|l}
\hline Intervent Neurol 2018;7:265-270 \\
\hline DOI: 10.1159/000486247 & $\begin{array}{l}\text { ○ 2018 S. Karger AG, Basel } \\
\text { www.karger.com/ine }\end{array}$ \\
\hline
\end{tabular}

Hu et al.: Diagnosis and Treatment of Acute Carotid Stent Thrombosis

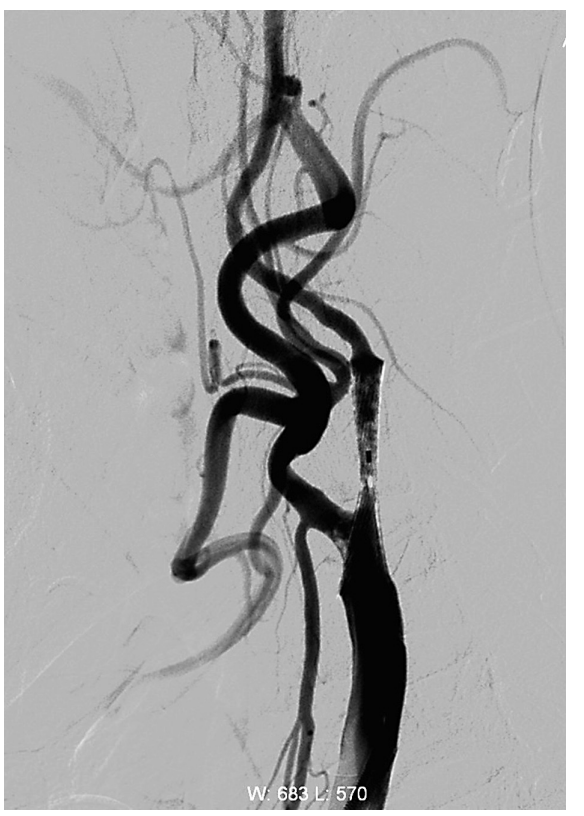

Fig. 4. Arterial thrombolysis was performed by a microcatheter in a carotid stent.

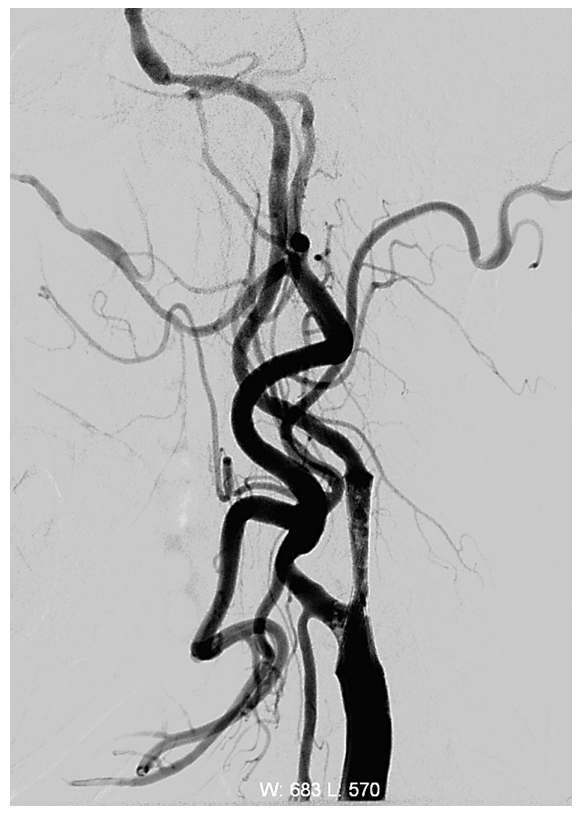

Fig. 5. The thrombus was not completely dissolved.

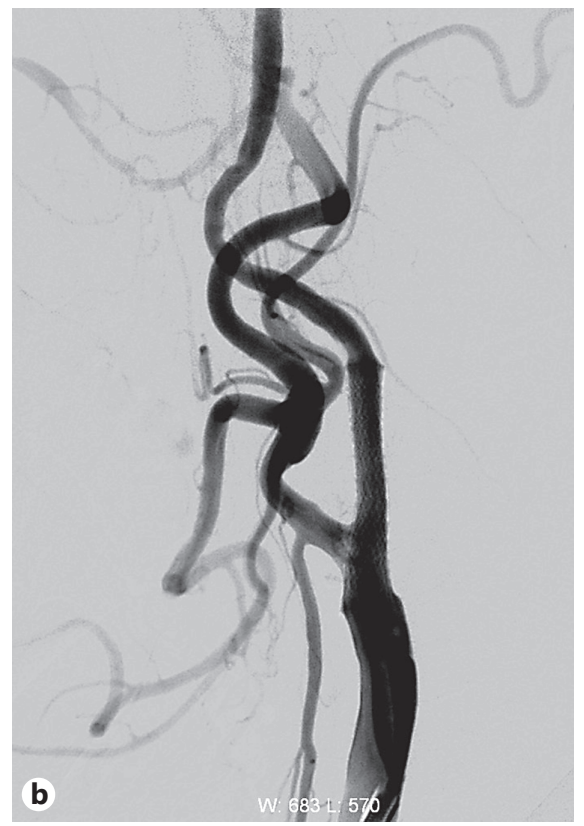

Fig. 6. a, b Final angiogram showing complete dissolution of the thrombus.

In our case, the patient without coagulation abnormality was administered aspirin and clopidogrel daily before the CAS; he showed no clopidogrel resistance. Thus, the probable cause of ACST was that the carotid stent failed to completely stick to the lining of the blood vessel, which led to excessive residual stenosis. Vascular endothelial injury may be another probable reason. 
Fig. 7. Duplex ultrasound showing good stent apposition and normal in-stent velocities at the 10-month follow-up.

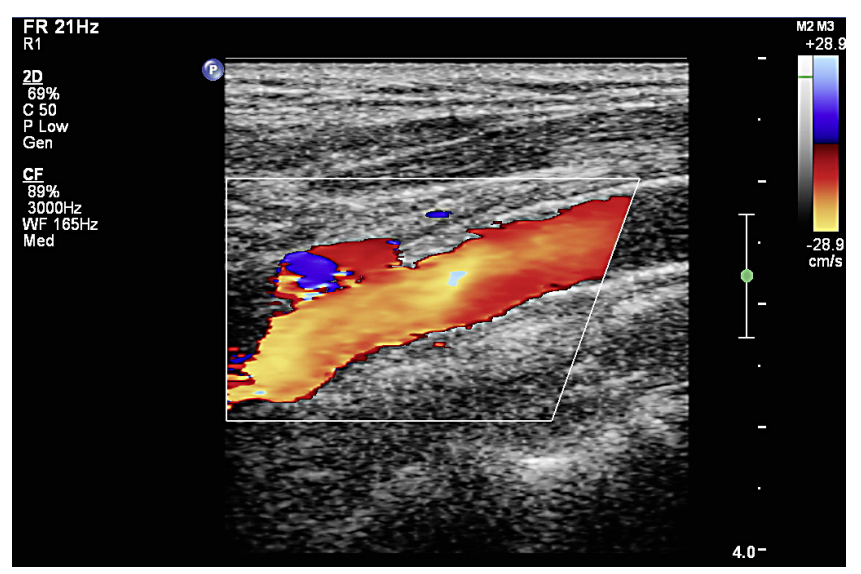

Early and rapid diagnosis and treatment of ACST was crucial for the improvement of the patient's outcome. During the CAS procedure, we adopted a "wait-and-see" strategy. i.e., observation of vascular appearances for a few minutes after carotid stent deployment. When carotid artery occlusion occurred during the wait-and-see period, the probable cause was immediately identified by selective angiography without retrieval of the guiding catheter or sheath. The proper treatment method was selected timely according to the angiogram results. Thus, it is probably a feasible method to ensure early and rapid diagnosis of ACST.

In papers published after 2000, in which some effective treatment strategies were listed, only 19 cases of ACST were reported [3-5]. The proper methods depended largely on the neurologists' experience, thrombotic state, and the intervention materials available. Though both mechanical thrombectomy and cerebral endarterectomy were effective therapies for ACST [3], it may be not the case for this patient due to more serious complications triggered by possible overuse of antiplatelet medication.

The forced arterial suction thrombectomy technique using the Penumbra System reperfusion catheter was an alternative approach for the management of ACST [3-5] and was mainly used for recanalization of acute occlusion of the major cerebral artery. However, it remains unclear whether it can resolve thrombi caused by plaque protrusion.

Another strategy mentioned in the treatment of ACST included systemic or local administration of a glycoprotein IIb-IIIa inhibitor or recombinant tissue plasminogen activator [3-5]. However, when the thrombus was not completely dissolved, the mechanical technique (i.e., percutaneous angioplasty or stent-in-stent) was superior to thrombolytic agents for the treatment of ACST. In our case, ACST was attributed to poor stent adherence. Artery thrombolysis partly dissolved the in-stent thrombosis temporally, and balloon redilation was capable of opening circumferentially to displace the thrombus, restoring the blood flow in the occluded vessel. Thus, the stent adhered completely to the lining of the blood vessel. Once antegrade flow was restored, endogenous thrombolysis could dissolve the thrombus.

In conclusion, ACST is a very rare complication of CAS and can have devastating effects on the survival of the patient. For ACST when the stent does not fully adhere to the blood vessel, a mechanical approach should be a feasible solution to the problem.

\section{Disclosure Statement}

The authors have no conflicts of interest to declare. This research received no specific grant from any funding agency in the public, commercial, or not-for-profit sectors. 


\section{References}

1 Brott TG, Hobson RW 2nd, Howard G, Roubin GS, Clark WM, Brooks W, Mackey A, Hill MD, Leimgruber PP, Sheffet AJ, Howard VJ, Moore WS, Voeks JH, Hopkins LN, Cutlip DE, Cohen DJ, Popma JJ, Ferguson RD, Cohen SN, Blackshear JL, Silver FL, Mohr JP, Lal BK, Meschia JF; CREST Investigators: Stenting versus endarterectomy for treatment of carotid-artery stenosis. N Engl J Med 2010;363:11-23.

2 Okazaki T, Satomi J, Satoh K, Hirasawa M, Nagahiro S: Rescue revascularization therapy with a stent-in-stent technique for acute intracranial internal carotid artery occlusion. Neurol Med Chir (Tokyo) 2005;45:253-258.

3 Markatis F, Petrosyan A, Abdulamit T, Bergeron P: Acute carotid stent thrombosis: a case of surgical revascularization and review of treatment options. Vascular 2012;20:217-220.

4 Steiner-Böker S, Cejna M, Nasel C, Minar E, Kopp CW: Successful revascularization of acute carotid stent thrombosis by facilitated thrombolysis. Am J Neuroradiol 2004;25:1411-1413.

5 Iancu A, Grosz C, Lazar A: Acute carotid stent thrombosis: review of the literature and long-term follow-up. Cardiovasc Revasc Med 2010;11:110-113. 